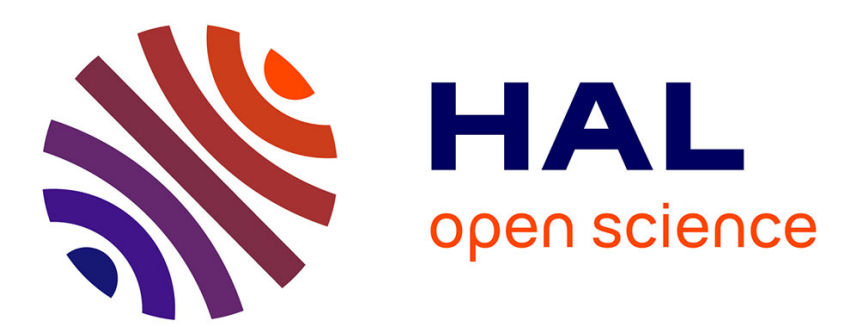

\title{
Optimization of an MEMS Magnetic Thin Film Vibrating Magnetometer
}

T. Perrier, R. Levy, B. Bourgeteau-Verlhac, P. Kayser, J. Moulin, S. Paquay

\section{To cite this version:}

T. Perrier, R. Levy, B. Bourgeteau-Verlhac, P. Kayser, J. Moulin, et al.. Optimization of an MEMS Magnetic Thin Film Vibrating Magnetometer. IEEE Transactions on Magnetics, 2016, 53 (4), pp.1-5. 10.1109/TMAG.2016.2622480 . hal-01743409

\section{HAL Id: hal-01743409 \\ https://hal.science/hal-01743409}

Submitted on 26 Mar 2018

HAL is a multi-disciplinary open access archive for the deposit and dissemination of scientific research documents, whether they are published or not. The documents may come from teaching and research institutions in France or abroad, or from public or private research centers.
L'archive ouverte pluridisciplinaire HAL, est destinée au dépôt et à la diffusion de documents scientifiques de niveau recherche, publiés ou non, émanant des établissements d'enseignement et de recherche français ou étrangers, des laboratoires publics ou privés. 


\title{
Optimization of a MEMS Magnetic Thin Film Vibrating Magnetometer
}

\author{
T. Perrier ${ }^{1,2}$, R. Levy ${ }^{1}$, B. Bourgeteau-Verlhac ${ }^{1}$, P. Kayser ${ }^{1}$, J.Moulin ${ }^{2}$, S. Paquay ${ }^{3}$ \\ ${ }^{1}$ ONERA - The French Aerospace Lab, 92320 Châtillon, France \\ ${ }^{2}$ Institut d'Electronique Fondamentale, UMR 8622, Université Paris-Sud, 91405 Orsay, France \\ ${ }^{3}$ Open Engineering S.A., Rue Bois Saint Jean 15/1, B-4102 Seraing, Belgium
}

\begin{abstract}
This paper presents models developed through analytical or numerical computation and finite element analysis to improve the resolution of a new kind of vibrating magnetometers. This peculiar magnetometer uses the piezoelectric transduction to actuate a quartz resonator at its resonance frequency taking advantage of the high $Q$ factor of a quartz resonator to achieve high resolution. The magnetic sensitive element is a thin ferromagnetic film of Nickel-Cobalt which is sputtered on the moving beams of the resonator. This magnetic thin film applies a periodic torque on the resonator, shifting its resonance frequency. This torque depends on the magnetic field applied; therefore the value of the magnetic field can be deduced from the frequency shift measurement. The aim of this paper is to develop and improve sensitivity models which will be useful tools in a future work to establish the optimal geometry for a resonator and the best position of the magnetic thin film on it in order to improve the sensitivity and resolution of the global sensor.
\end{abstract}

Index Terms - Ferromagnetic thin film, MEMS, quartz resonator, sensitivity modeling, magnetometer

\section{INTRODUCTION}

$\mathrm{R}$ ecent development of high resolution magnetometers have enabled new applications such as indoor navigation [1] or Unattended Ground Sensors (UGS) for vehicle detection and identification [2], [3]. These applications need magnetometers showing high resolutions $(<10 \mathrm{nT})$, compactness $\left(<1 \mathrm{~mm}^{3}\right)$ and low power consumption $(<1 \mathrm{~mW})$. The most suitable technology for these applications is Anisotropic Magneto Resistance (AMR) magnetometer because of their high resolution and effective industrial process but because of their non-linear sensitivity they need a long calibration process before their use or even in use [1]. During the last decade, MEMS magnetometers have been developed and use most of the time two operating principles. The most common principle uses the Lorentz force induced on a moving structure where a current is circulating [4]-[9]. Another actuation principle uses the magnetic torque induced on a ferromagnetic layer deposited on a moving structure when its magnetization is not aligned with the external magnetic field [10]-[13]. These magnetometers meet the compactness and power consumption requirements but their resolution needs to be improved.

Among these, magnetic thin film vibrating beam MEMS magnetometer [14], [15] consist in a thin film of hard ferromagnetic material, NiCo for this study, sputtered on a cantilever with a magnetic moment aligned along the length of the cantilever. As the resonator vibrates under a magnetic field, the magnetostatic interaction produces a mechanical moment on the cantilever that stiffens its spring constant thus shifting its resonance frequency linearly with the magnetic field applied. Magnetic field value can be deduced through the measure of this frequency shift. Indeed, this frequency shift depends linearly on the value of the magnetic field applied [16].

In order to optimize this new kind of MEMS magnetometer, this paper presents a behavioral model including the resonator, the mechanical torque induced by the thin film on the resonator as well as the oscillator circuit and the frequency counter which provides access to sensitivity of multiple sensors geometries. Moreover a Finite Element Method (FEM) which simulates the sensitivity of the sensor as a function of the geometry of resonators and the position and properties of a ferromagnetic layer is also presented. The model developments are presented as well as their validation with four magnetometer prototypes and with an analytical model of the sensitivity developed in a previous paper [16]. These models will enable to investigate the advantages of various shapes of resonators as well as the optimization of the thin film position on the resonator, of the oscillator circuit and the magnetometer as a whole. The advantages of both behavior and finite element models are also discussed.
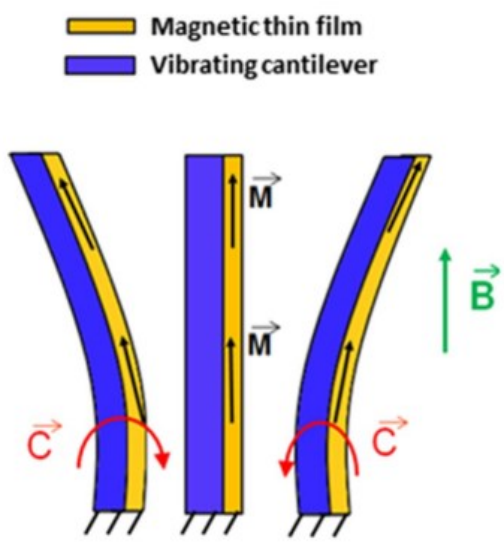

FIG. 1: Principle of operation of the vibrating cantilever magnetometer (from [16])

\section{BEHAVIORAL MODEL DEVELOPMENT}

\section{A. Resonator}

The resonator behavioral model implemented in Verilog-A language uses the NODAS library [17]. It is a purely 


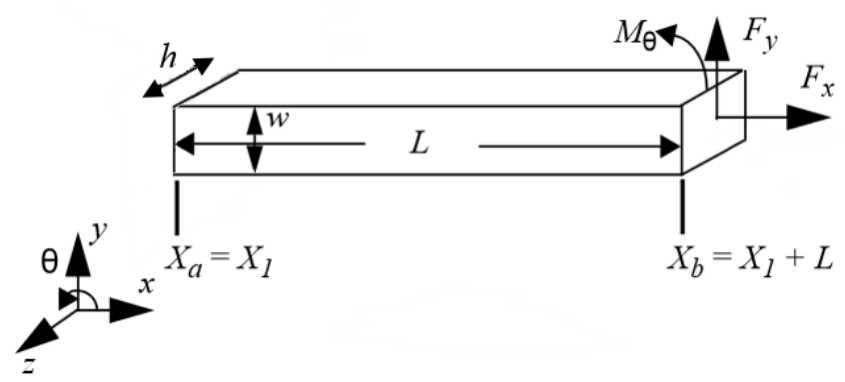

FIG. 2: Schematic of an elementary beam

mechanical model implementing Euler-Bernoulli equations which characterize the motion of beam under different strengths, moments and boundaries conditions. This is a homogeneous and isotropic model that enables the simulation of a beam motion in the $x-y$ plane only due to translational and rotational displacements $(\delta \mathrm{x}, \delta \mathrm{y}, \delta \theta)$, forces $\left(\mathrm{F}_{\mathrm{x}}, \mathrm{F}_{\mathrm{y}}\right)$ and moment $\left(\mathrm{M}_{\theta}\right)$ applied to its two ports as shown in Fig. 2. In order to include in the model the ability to modify the shape of the resonator and to deposit the magnetic thin film locally on the resonator, the model is composed of many elementary beams linked to each other where, if needed, it is possible to apply separately external strengths and moments.

\section{B. Magnetic thin film}

The mechanical torque induced by the magnetostatic interaction between the magnetic thin film and the external magnetic field at position $\mathrm{x}$ on the cantilever is:

$$
\tau(x)=A \Delta x M_{r} B \sin (\theta(x))
$$

Where $A$ is the area of the cross-section of the thin film, $\Delta x$ the length of the elementary portion of the beam where the thin film is deposited, $M_{r}$ the remanent magnetization of the film, $B$ the external magnetic field along $\mathrm{x}$-axis, and $\theta$ the local angle between the beam and the $\mathrm{x}$-axis.

The thin film model consists in a Verilog-A element that takes as input the angular displacement $\theta$ at a node between two elementary beams and sends as output the mechanical moment $\tau$ at the same node, as shown in Fig. 3. This implementation enables to simulate the behavior of a sensor with the possibility to modify easily the portion of the sensor where the magnetic thin film is deposited. Indeed, with the graphical interface of the Cadence ${ }^{\circledR}$ software which allows to do Verilog-A simulation, a mere box modeling (1) is connected at the node between all elementary beams where the magnetic thin film is supposed to be.

\section{Electric circuit}

The electric circuit consists in a self-sustained oscillator to drive the resonator at resonance and a frequency counter to measure the frequency shift induced by the magnetostatic interaction between the magnetization of the thin film and the external magnetic field. Both circuits are implemented in Verilog-A.

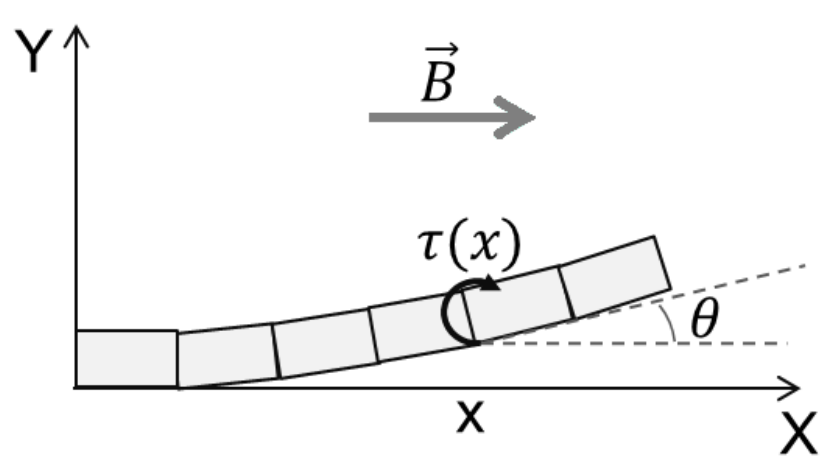

FIG. 3: Schematic of the whole beam composed of elementary beams and the mechanical moment induced by a thin film deposited locally on top of the beam.

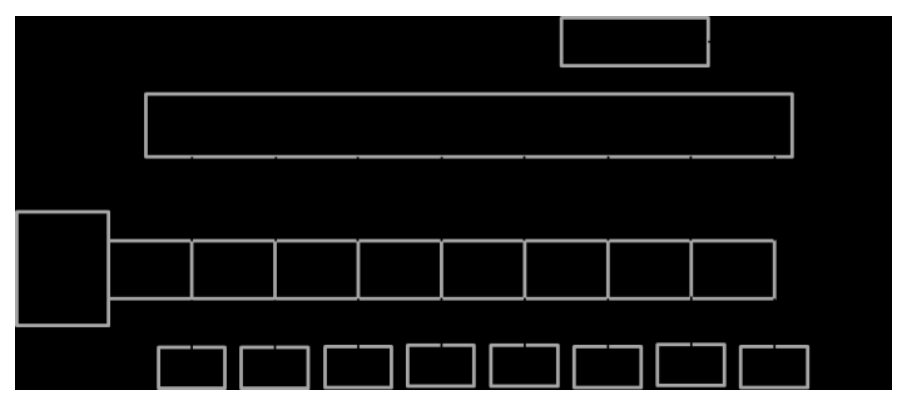

FIG. 4: Schematic of the magnetometer including the beam composed of elementary beams and anchored on one side, the magnetic thin film all along the beam length, the oscillator circuit to drive the resonator at resonance and the frequency counter to measure the oscillator frequency.

The oscillator takes as input the displacement at a node of the beam, applies a delay and an amplification to account for the Barkhausen criteria, then a soft clipper allows the limitation of the oscillation amplitude, and as output strength is applied at a beam node.

\section{FINITE ELEMENT MODEL}

\section{A. Implementation}

Assuming small displacements in the case of this study, (1) can be simplified and written as:

$$
\tau(x)=A \Delta x M_{r} B \theta(x)
$$

The implementation of volume and surface torque elements supporting (2) was successfully performed and validated in OOFELIE::Multiphysics $($ solver. These new elements permits to translate rigorously a volume/surface torque contribution into nodal forces contributions of 3D elements that consider only nodal displacements as degrees of freedom (no rotation degrees of freedom). The applied torque depends linearly on the displacement field itself, the new elements implementation introduces contributions in stiffness matrix of 


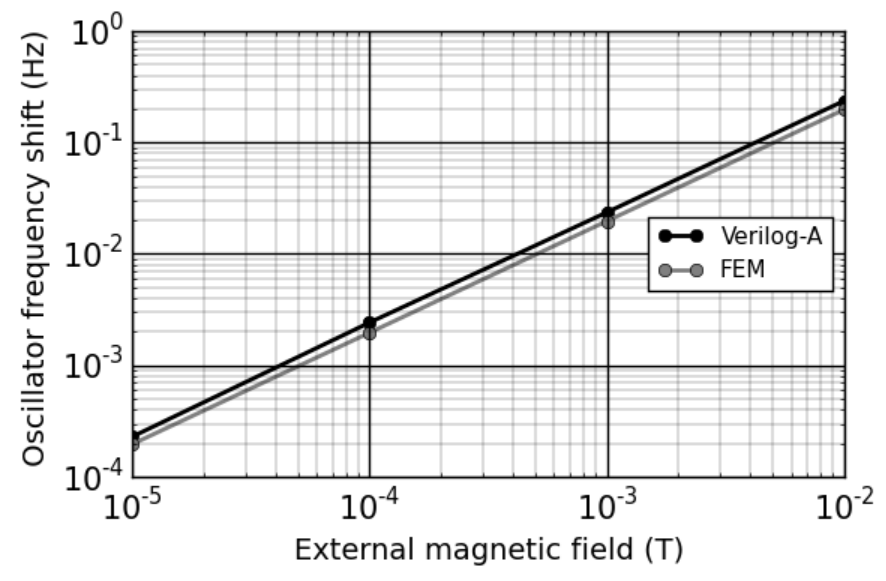

FIG. 5: Frequency shift versus magnetic field applied

the resulting FEM system.

\section{B. Simulation sensitivity}

To deduce sensitivity with FEM simulations, two modals computations are needed. The first one is performed without magnetic field, which provides the reference frequency and information on the deformation of the resonator. Indeed, knowledge of the deformation $\theta(x)$, which is a priori unknown, is necessary to apply magnetic torque. The second modal computation takes into account the magnetic torque contribution in stiffness matrix. The sensitivity of the sensors $\mathrm{K}_{\mathrm{B}}$ is then deduced from the frequency shift $\Delta \mathrm{f}$ between both simulations and the value of the magnetic field $\mathrm{B}$ applied:

$$
K_{B}=\frac{\Delta f}{B}
$$

\section{MODELS VALIDATION}

\section{A. Linear sensitivity}

First of all, the linearity between the frequency shift and the external magnetic field applied has been verified for both models. In Fig. 5, simulations are made for a simple clampedfree beam, fully covered on its top and laterally by a thin ferromagnetic film. It is assumed that the remanent magnetization $M_{R}$ of the thin layer along the beam is a typical value $10^{6} \mathrm{~A} / \mathrm{m}$ and its thickness $t$ is $1 \mu \mathrm{m}$. The length $L$ of the beam is $1400 \mu \mathrm{m}$, its width $w$ is $70 \mu \mathrm{m}$ and its thickness $h$ is $30 \mu \mathrm{m}$. Fig. 5 proved the linear sensitivity of this kind of magnetometer, which is advantageous in comparison to AMR magnetometers. With this shape, the sensitivity, i.e. the slope of $\Delta f$ vs. $B$, is $19.7 \mathrm{~Hz} / \mathrm{T}$ for FEM model and $23.6 \mathrm{~Hz} / \mathrm{T}$ for the Verilog-A model. The R-squared coefficient assuming a linear model for respectively FEM and Verilog-A models is of 1-3e6 and 1-1e-8 which proved the linear sensitivity of both models. R-squared for Verilog-A model is better because it is a linear model assumed in its implementation.

\section{B. Comparison to analytical model}

An analytical model was developed in a previous paper

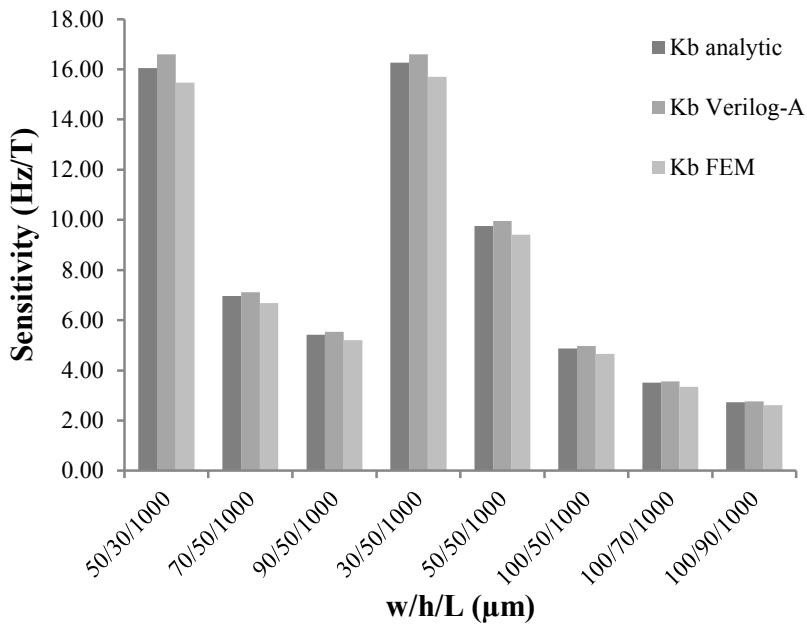

FIG. 6: Comparison of analytical, Verilog-A and FEM sensitivity model for different beams shape with $t=1 \mu \mathrm{m}$.

[16]. This model gives the sensitivity for a resonator with a thin film deposited on the top of a clamped-free beam vibrating at its first flexural mode:

$$
K_{B}=\frac{\Delta f}{B}=0.38 \frac{M_{R}}{\sqrt{E \rho}} \frac{1}{w} \frac{t}{h}
$$

where $\mathrm{E}$ and $\rho$ are respectively the Young modulus and the density of the vibrating beam.

Fig.6 compares the computed sensitivity of analytical, Verilog-A versus FEM models. Results show a very good agreement between all models when sensitivity is calculated in Bernoulli assumptions proving the relevant behavior of Verilog-A and FEM models. Most of the times, vibrating beams are considered in Bernoulli assumptions when deformations are small and when the cross-sectional dimensions of the beam, $\mathrm{h}$ and $\mathrm{w}$, are both 5 times smaller than its length L. Both assumptions are true in this study. It is clear in Fig. 7 that when calculations are made for beam shapes which do not respect Bernoulli assumptions, results between models diverge sharply. This divergence is easily understandable because analytical and Verilog-A models are developed under Bernoulli assumptions unlike FEM model.

FEM is a more accurate and general model; it can compute the true sensitivity for all resonator shapes and vibration modes. Moreover, FEM takes into account anisotropy of quartz that cannot be done with the Verilog-A model. Furthermore, preexisting developments allow the simulation of sensors with a multiphysics approach, taking into account thermal, electrical and mechanical effect and their coupling. Therefore, FEM model will be the main model to design sensors and study their behavior the most accurately. However, even if Verilog-A model is limited, it takes into account the whole electronic circuit of the sensor that is a big advantage for a global simulation. In fine, the Verilog-A model will be useful to simulate the global sensor with a simplified model for the resonator shape while FEM model 


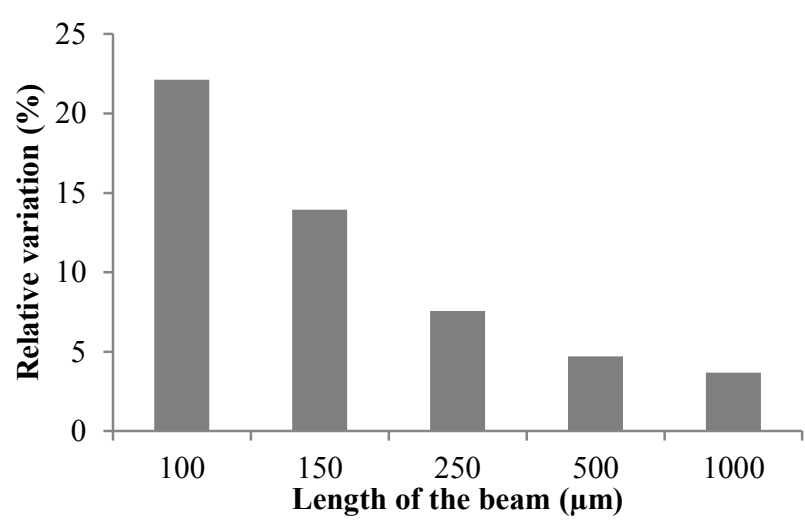

FIG. 7: Relative variation of calculated sensitivity between FEM and analytical model vs $L$ with $t=1 \mu \mathrm{m}$ and $\mathrm{w}=\mathrm{h}=50 \mu \mathrm{m}$ for beam that do not respect Bernoulli assumptions.
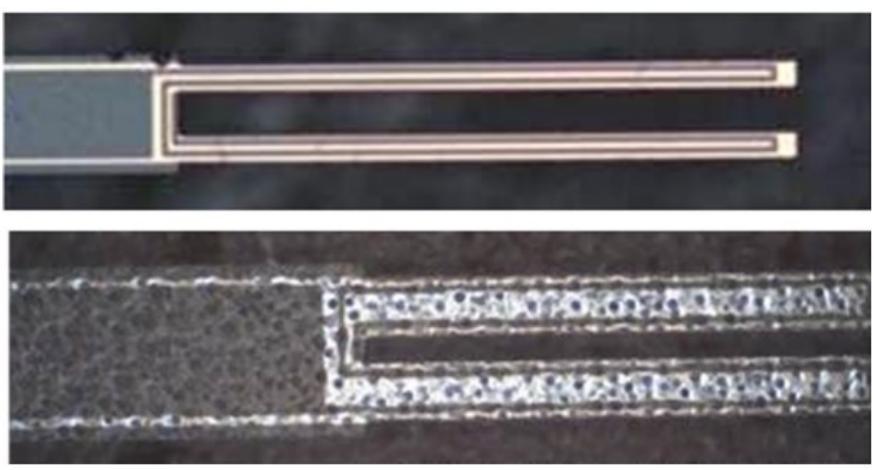

FIG. 8: Realized tuning fork: the actuation electrodes side and the deposited thin film on the other side (down) (from [16]).

will permit to study more complex geometries.

\section{Experimental validation}

These simulated sensitivities are then compared on sensitivity measured on four magnetometer prototypes. For the last three decades, quartz resonators are used in inertial and frequency/time measurements[18]. This study is directly derived of these previous studies. Here, magnetometers prototypes use piezoelectric transduction to actuate quartz resonators at their resonance frequency. Wafers used are in zcut with beams along the $y$ crystallographic axis. A system of 3 electrodes actuates resonators in a flexural mode using the piezoelectric effect of quartz.

Prototypes are simple tuning-fork resonator (cf. Fig. 8); on one side, electrodes actuate the tuning-fork, on the other side a $\mathrm{Ni}_{85} \mathrm{CO}_{15}$ thin layer is deposited by a RF diode sputtering system. As the thickness of beam is relatively small $(\sim 30 \mu \mathrm{m})$, RF-sputtering gives conformal coating, i.e. there is NiCo thin layer on top and lateral faces of beams. In order to measure their sensitivity, prototypes were inserted into a coil in a vacuum chamber. The oscillator frequency of the electric circuit is then measured with and without magnetic field to measure the sensor sensitivity (cf. Fig. 9)[16]. Measured sensitivities and corresponding simulations results are

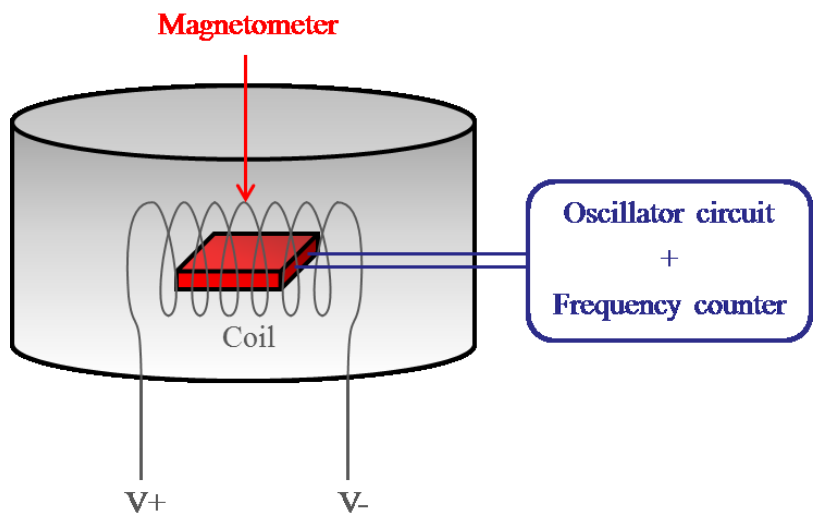

FIG. 9: Illustration of the experimental setup in a vacuum chamber

\begin{tabular}{cccccc}
\hline \hline $\begin{array}{c}\text { Thin Film } \\
\text { Thickness t } \\
(\mathrm{nm})\end{array}$ & $\begin{array}{c}\text { Beam } \\
\mathrm{w} / \mathrm{h} / \mathrm{L} \\
(\mu \mathrm{m})\end{array}$ & Experiment & Analytic & Verilog-A & FEM \\
\hline 200 & $80 / 30 / 1000$ & 5 & 4 & 4 & 4 \\
500 & $50 / 30 / 800$ & 17 & 20 & 20 & 16 \\
500 & $70 / 30 / 1400$ & 12 & 12 & 12 & 10 \\
1000 & $70 / 30 / 1400$ & 19 & 24 & 23 & 20 \\
\hline \hline
\end{tabular}

Table 1: Comparison of experimental and theoretical sensitivity values for different beam shapes and models.

presented in Table 1. Assuming in models that the thin layer is also deposited on lateral faces of beams, which is confirmed by microscope observations, results are in good agreement with all models. However, measurements were carried out with only one magnetic field value of $+/-1$ Gauss. In future work on new kind of resonators, it will be interesting to prove the linearity experimentally on a large range of magnetic field value.

\section{MODELS EXPLOITATION}

Depositing of a thin film on a vibrating structure induces viscoelastic losses by frictions in the thin film that decreases the total quality factor of the resonator and as a consequence the resolution of the magnetometer [19]-[22]. In this study, the origins of the losses can be either of the electrodes or ferromagnetic film.

This additional damping occurs mainly near the anchor of the beam where mechanical stress is high, so it would be advantageous to deposit the magnetic thin film only near the tip of the beam where mechanical stress is low and deflection is even so high. The models simulations enable to predict the sensitivity variation as a function of the ferromagnetic film position on the beam as shown in Fig. 10. It is clear that the thin film can be deposited only on the half of the beam without decreasing too much the sensitivity. 


\section{REFERENCES}

[1] K. Mohamadabadi, "Anisotropic magnetoresistance Magnetometer for inertial navigation systems ECOLE POLYTECHNIQUE Anisotropic Magnetoresistance Magnetometer for inertial navigation systems by A thesis submitted in partial fulfillment of the," école Polytechnique, 2014.

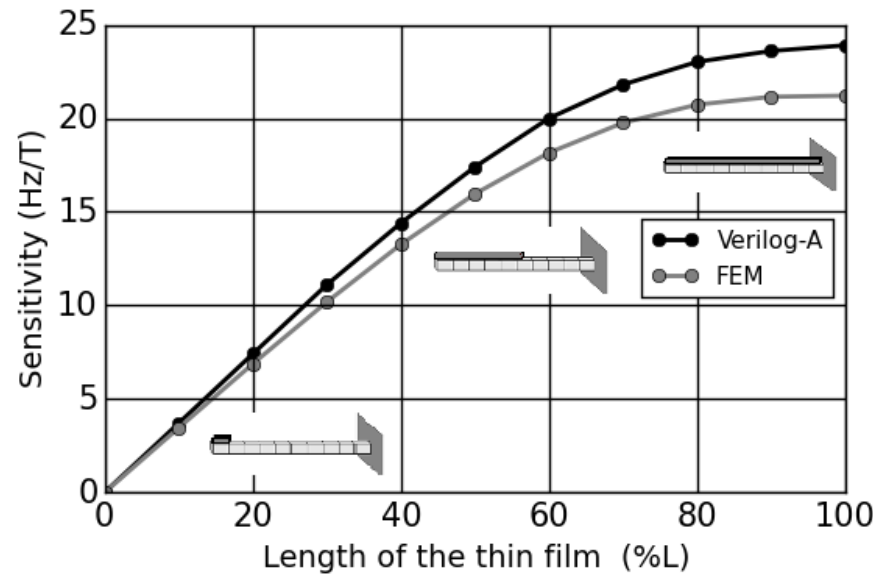

\section{FIG. 10: Simulated sensitivity for various position of the thin film on a beam with $L=1400 \mu \mathrm{m}, w=70 \mu \mathrm{m}, h=30 \mu \mathrm{m}$ and $\mathrm{t}=1 \mu \mathrm{m}$.}

\section{CONCLUSION}

A behavioral model of the magnetic thin film vibrating beam magnetometer has been developed and implemented in Verilog-A language; it includes the resonator, the mechanical torque induced by the thin film as a function of the external magnetic field, the oscillator circuit and the frequency counter. A FEM model has also been developed to take into account magnetic torque on vibrating structure. Simulations of this model have been compared with experimental measurements on magnetometer prototypes for validation. FEM model is obviously the best model to design sensors with a multiphysic approach that enables to study complex thermo-electromechanical coupling. In fine Verilog-A will be useful to study the whole sensor with a simplified geometry with the electronic circuit associated. These models allow in future work the investigation of the advantages of various resonator shapes as well as the optimization of the position of the deposited thin film on the resonator. Future work will also be related to on the one hand the calculation of the damping terms to complete the model and enable the simulation of the signal to noise ratio[16], and on the other hand the implementation of various materials (silicon/quartz) and various transduction techniques (electrostatic/piezoelectricity) in a global model in order to optimize the magnetometer performances.

\section{ACKNOWLEDGMENT}

This work was financially supported by the French Ministry of Defense (DGA). 2010 13th Int. Conf. Inf. Fusion, pp. 1-8, 2010.

J. Fine, A. Edelstein, and D. Hull, "AMR Magnetometer Data on Moving Military Vehicles at Aberdeen Proving Ground," Army Research Laboratory, 2007.

H. T. D. Grigg and B. J. Gallacher, "Xylophone Bar Magnetometry and Inertial-Grade MEMS Optimisation : A Multiphysics Approach," pp. 1-7, 2011.

D. H. Ren, M. Y. Cui, L. Q. Wu, T. W. Guo, C. T. Xu, and Z. You, "A MEMS torsional resonant magnetometer for the attitude determination in space," in 2013 Transducers \& Eurosensors XXVII: The 17th International Conference on Solid-State Sensors, Actuators and Microsystems (TRANSDUCERS \& EUROSENSORS XXVII), 2013, no. June, pp. 462-465.

M. J. Thompson, M. Li, and D. A. Horsley, "Low power 3-axis Lorentz force navigation magnetometer," in 2011 IEEE 24th International Conference on Micro Electro Mechanical Systems, 2011, no. 3, pp. 593-596.

[7] R. Sunier, T. Vancura, Y. Li, K.-U. Kirstein, H. Baltes, and O. Brand, "Resonant Magnetic Field Sensor With Frequency Output," Microelectromechanical Syst. J., vol. 15, no. 5, pp. 1098-1107, 2006.

[8] M. Li, V. T. Rouf, M. J. Thompson, and D. A. Horsley, "Three-Axis Lorentz-Force Magnetic Sensor for Electronic Compass Applications," J. Microelectromechanical Syst., vol. 21, no. 4, pp. 1002-1010, Aug. 2012.

[9] J. Kyynäräinen, J. Saarilahti, H. Kattelus, A. Kärkkäinen, T. Meinander, A. Oja, P. Pekko, H. Seppä, M. Suhonen, H. Kuisma, S. Ruotsalainen, and M. Tilli, "A 3D micromechanical compass," Sensors Actuators A Phys., vol. 142, no. 2, pp. 561-568, Apr. 2008.

[10] H. H. Yang, N. V. Myung, J. Yee, D.-Y. Park, B.-Y. Yoo, M. Schwartz, K. Nobe, and J. W. Judy, "Ferromagnetic micromechanical magnetometer," Sensors Actuators A Phys., vol. 97-98, pp. 88-97, Apr. 2002.

[11] D. Guangtao, C. Xiangdong, L. Qibin, L. Hui, and G. Huihui, "MEMS magnetic field sensor based on silicon bridge structure," $J$. Semicond., vol. 31, no. 10, p. 104011, Oct. 2010.

[12] X. Yin, Q. Jiao, L. Yuan, and S.-H. Liou, "MEMS Torsion Oscillator Magnetic Field Sensor," IEEE Trans. Magn., vol. 49, no. 7, pp. 3890-3892, Jul. 2013.

[13] D. Ettelt, P. Rey, M. Savoye, C. Coutier, M. Cartier, O. Redon, M. Audoin, A. Walther, P. Robert, Y. Zhang, F. Dumas-Bouchiat, N. M. Dempsey, and J. Delamare, "A new low consumption 3D compass using integrated magnets and piezoresistive nano-gauges," in 2011 16th International Solid-State Sensors, Actuators and Microsystems Conference, 2011, pp. 40-43.

[14] R. Levy, P. Kayser, S. Masson, B. Bourgeteau, and J. Moulin, "A miniature vibrating cantilever magnetometer," in 2015 Symposium on Design, Test, Integration and Packaging of MEMS/MOEMS (DTIP), 2015, pp. 1-4.

[15] J. W. van Honschoten, W. W. Koelmans, S. M. Konings, L. Abelmann, M. Elwenspoek, J. van Honschoten, W. W. Koelmans, and J. W. van Honschoten, "Nanotesla torque magnetometry using a microcantilever," Proc. Eurosensors XXII, Eur. Conf. Solid-State Transducers, pp. 597-600, 2008.

[16] R. Levy, T. Perrier, P. Kayser, B. Bourgeteau, and J. Moulin, "A micro-resonator based magnetometer," Microsyst. Technol., Jan. 2016.

[17] G. K. Fedder and Q. Jing, "NODAS 1.3 - Nodal Design Of Actuators And Sensors," in Proceedings of 1998 IEEE/VIUF international workshop on behavioral modeling and simulation, 1998.

[18] O. Le Traon, D. Janiaud, J. Guerard, R. Levy, S. Masson, O. Ducloux, M. Pernice, and R. Taibi, "The fairy world of quartz vibrating MEMS," in 2012 European Frequency and Time Forum, 2012, pp. 214-220.

[19] A. Frangi, M. Cremonesi, A. Jaakkola, and T. Pensala, "On the
C. T. Christou and G. M. Jacyna, "Vehicle detection and localization using Unattended Ground Magnetometer Sensors," 
optimization of piezoelectrically actuated MEMS resonators," in 2012 IEEE International Ultrasonics Symposium, 2012, pp. $1043-$ 1046.

[20] O. Le Traon, S. Masson, C. Chartier, and D. Janiaud, "LGS and GaPO4 piezoelectric crystals: New results," Solid State Sci., vol. 12, no. 3, pp. 318-324, Mar. 2010.

[21] B. Bourgeteau-Verlhac, R. Levy, T. Perrier, P. Lavenus, J. Guerard, and O. Le Traon, "Gold thin film viscoelastic losses of a length extension mode resonator," in 2016 European Frequency and Time Forum (EFTF), 2016, pp. 1-4.

[22] I. Dufour, F. Lochon, S. M. Heinrich, F. Josse, and D. Rebiere, "Effect of Coating Viscoelasticity on Quality Factor and Limit of Detection of Microcantilever Chemical Sensors," IEEE Sens. J., vol. 7, no. 2, pp. 230-236, Feb. 2007. 